\title{
Development of RNAi-Based Vector Aims at Creating Antiviral Soybean Plants in Vietnam
}

\author{
Lo Thi Mai Thu, Le Van Son, Chu Hoang Ha, and Chu Hoang Mau
}

\begin{abstract}
Soybeans (Glycine max (L.) Merrill) are easily infected by soybean mosaic virus (SMV) and bean yellow mosaic virus (BYMV), which may reduce their yields from $66 \%-80 \%$. At present, preventive measures are still the main methods to reduce losses caused by these two viruses. RNAi is thus considered as a modern and promising technique to generate plants resistant to viruses. In this study, we cloned and characterized the Coat protein (CP) coding gene of SMV cultivar SL1 and SL2, which is $720 \mathrm{bp}$ in length and encodes 240 amino acids. The two conserved regions of $\mathrm{CP}$ gene from these two viruses (SMV and BYMV) were integrated into a 573 bp fragment, called CPi(SMV-BYMV). Vector pK7GW-CPi (SMV-BYMV), carrying the CPi (SMV-BYMV) fragment in sense and antisense orientation separated by an intron, was successfully designed by Gateway technology (Invitrogen). Once being transformed into plant cells, this vector is expected to be transcribed into a hairpin RNA structure that could induce RNAi mechanism to degrade the CP genes of SMV and BYMV, thus limit their infection. Therefore, this vector could be a potential tool to generate transgenic soybean plants that are resistant to both two virus species SMV and BYMV in Vietnam.
\end{abstract}

Index Terms-BYMV, CPi, CP gene, Glycine max, SMV, RNAi, transgenic vector.

\section{INTRODUCTION}

Soybeans (Glycine max (L.) Merrill) are industrial crops which have high economic and nutritional value, as well as as well as play an important role in improving soil fertility. Soybeans, however, are easy to be infected by numerous virus species (more than 100 species according to statistics) [1], in which some could cause severed damage such as soybean mosaic virus (SMV) and bean yellow mosaic virus (BYMV) [1], [2]. SMV and BYMV can simultaneously infect into a soybean plant and their symptoms are difficult to distinguish. Their simultaneous infection could reduce soybean yields from $66 \%-80 \%$ [3], [4]. SMV and BYMV can be spread by aphids, in addition to longer diseases transmitted through seeds.

At present, main methods to reduce losses caused by these two viruses are still tradditional preventive measures, such as selection of the disease-resistant plants, field sanitation and crop rotation. These traditional methods are often

Manuscript received December 6, 2013; revised March 21, 2014

Lo Thi Mai Thu is with the Department of Biology, Taybac University, Vietnam (e-mail: thubiotbu@gmail.com).

Le Van Son and Chu Hoang Ha are with the Department of Plant Cell Biotechnology, Institute of Biotechnology, VAST, Vietnam.

Chu Hoang Mau is with the Department of Genetics and modern Biology, faculty of Biology, Thainguyen University, Vietnam (e-mail: mauchuhoang@gmail.com). complicated, time consuming, ineffective and unsustainable. Therefore, scientists have to seek new efficient methods to control these viruses. One of the most potential approaches is RNA interference (RNAi). RNAi is a process that inhibits gene expression by the double stranded RNA (dsRNA) that can cause the degradation of target mRNA or blocking translation [5]-[7]. Based on this principle, some author has successfully applied RNAi mechanism in the strategy to create transgenic plants resistant to viruses [8]-[10]. In the past few year, RNAi has been broadly used as a new biotechnological tool for the control of virus diseases in plants.

Genomes of both SMV and BYMV consists of one single-stranded, linear, positive-sense RNA molecule. As in all members of the genus Potyvirus, their single open reading frame is expressed as a large polyprotein which is autoproteolytically cleaved to yield ten functional proteins: $\mathrm{P} 1$ proteinase $(\mathrm{P} 1)$, helper component proteinase (HC-Pro), Protein P3 (P3), $6 \mathrm{kDa}$ protein 1 (6K1), Cytoplasmic inclusion protein $(\mathrm{CI}), 6 \mathrm{kDa}$ protein $2(6 \mathrm{~K} 2)$, the viral genome linked protein (VPg), Nuclear inclusion protein $\mathrm{A}$ (NIA), Nuclear inclusion protein B (NIB), and coat protein (CP) [11], [12]. The Coat Protein plays vital roles in protection of the RNA inside, infection, movement of virus from cell to cell, transmission by aphids, and symptom formation [13]. Therefore, silencing the CP gene can suppress the infection of SMV and BYMV.

The RNAi-based vector containing CPi fragment inverted repeats could be used to transfer into plants and would express into hairpin RNA structure (hairpin RNA, hpRNA), thereby stimulating RNAi mechanism when there is penetration of SMV or BYMV into plants. Scientists noticed that the buffer zone of hpRNA be repeated with a intron sequences (ihpRNA) will inducing gene silencing with the highest level [14], [15]. Kenny et al. (2007) have created lines of transgenic pea plants antiviral bean golden mosaic virus with resistance ability up to $93 \%$ [16]. Lim et al. (2007) studied on HC-Pro gene and transferred into soybean plants has noticed that, when transgenic plants were infected SMV after 2 weeks, the leaf mosaic symptoms disappear and SMV content has decreased significantly, however HC-Pro gene of SMV has altered leaf morphology and reduce granulating in transgenic soybean plants [17]. Generally, most of the transgenic plants slow down the accumulation of virus and slow or reduce disease symptoms. However, the disease resistance of crops still heavily depends on the function and location of the transgene. In Vietnam, the first successful application of RNAi in generation of plants resistant to viruses was the generation of transgenic tobacco plants resistant to Cucumber mosaic virus (CMV) and Tobacco mosaic virus (TMV). The transgenic tobacco lines containing 
simultaneously two CP gene fragments of TMV and CMV showed resistance ability to two virus strains up to $60 \%$ [18]. Next was the success in creation of transgenic papaya plants resistant to papaya ringspot virus (PRSV) [19], [20]. However, the authors also found that the rates of disease resistance in transgenic plant lines depend on gene fragments selected for designing RNAi-based vector. Mosaic disease in soybean plant caused by SMV and BYMV have symptoms that are difficult to distinguish, and soybean plants can also be coinfected by these two viruses at the same time. Thus, in this study, we present some results on amplification of $\mathrm{CP}$ gene from mRNA isolated from SMV lines SL1, SL2 in Son La province, Vietnam. After that, we designed an RNAi-based vector carrying the conserved region of $\mathrm{CP}$ genes of both SMV and BYMV, with the desire to create transgenic soybean plants that are resistant to both these two virus species.

\section{MATERIALS AND METHODS}

\section{A. Materials}

Plant materials: Leaves of soybean plants which are suspected to be infected by SMV collected in several localities of Son La province, Vietnam were used for total RNA extraction of SMV. Tobaco cultivars N. tabacum K326 was supplied by Department of Plant Cell Technology, Institute of Biotechnology, Vietnam.

Bacteria strains: E.coli $\mathrm{DH} 5 \alpha$, E. coli One Shot ${ }^{\circledR}$ TOP 10 (Invitrogen) and strains $A$. tumefaciens CV58C1 carrying plasmid pGV2660 cytotoxic .

Plasmid: Vector cloning PBT, transgenic vector pK7GWIWG2(II), vector cloning pENTR $^{\mathrm{TM}}$ and pENTRTM Directional TOPO ${ }^{\circledR}$ Cloning KIT (Invitrogen), KIT Gateway Gateway $^{\circledR}$ LR reaction Clonase ${ }^{\mathrm{TM}}$ II.

\section{B. Methods for Cloning CP Gene and Synthesis of CPi Gene Region of SMV and BYMV}

Total RNA was isolated using Trizol Reagent KIT, cDNA was synthesized using the Maxima ${ }^{\circledR}$ First Strand cDNA Synthesis KIT. The cDNA was used as template for PCR to amplify coding region of $\mathrm{CP}$ gene of SMV. From the information of SMV CP gene sequence on GenBank, code number X63771, we designed the primer pair SMVcp-F/SMVcp-R to amplify the coding region of SMV CP gene:

\section{SMVcp-F: 5'-TTACCATCAGGCAAGGAGCAGGAAG-3'}

\section{SMVcp-R: 5'-TGGACTTGATGGGAACATCTCAACT-3'}

PCR products were then examined by electrophoresis on $1.0 \%$ agarose gel and purified by $\mathrm{JET}^{\mathrm{TM}}$ Gene Gel Extraction KIT. Recombinant vector was transformed into $E$. coli $\mathrm{DH} 5 \alpha$ cells with variable heat shock. Bacteria carrying recombinant vector were selected on antibiotic selective environment (carbenicilin, kanamycin). The colonies carrying the recombinant vector were selected by colony-PCR with specific primers, then cultured in LB supplemented with antibiotic to collect biomass. Recombinant plasmids were obtained by extracting using the molecular cloning methods
[21]. The two CP gene sequences of SMV lines SL1, SL2 were determined on the automated DNA ABI PRISM 3100 Avant Genetic Analyzer machine. It utilized the software biological Clustal to test the sequence of CP gene.

From the sequences of $C P$ genes of SMV and BYMV, we identified their conserved regions using BLAST in NCBI. Primer pair SMV-CPi-F/SMV-CPi-R was used to integrate these two regions from these two viruses:

MV-CPi-F: 5'- CACCGCAGCAGAAGCTTACA -3' BYMV-CPi-R: 5'- ATGTTCCGAACCCCAAGCAA-3'

\section{Methods of Designing Transgenic Vector Carrying RNAi Structure Containing CPi Gene Region by Gateway Technique}

Designing of transgenic vector in plants carrying RNAi structures is performed as described by Karimi et al. (2002) [10].

Fragment CPi (SMV-BYMV) is amplified by PCR with primer pair MV-CPi-F/BYMV-CPi-R. PCR products were examined by electrophoresis on $1.0 \%$ agarose gel and purified by GeneJET PCR Purification Kit (Thermo Scientific). The CPi fragment was then cloned into vector $\mathrm{pENTRY/D} \mathrm{to} \mathrm{create} \mathrm{recombinant} \mathrm{vector} \mathrm{pEN}-\mathrm{CPi}$ containing the recombinant position attL (Recombinant position with attrR on vector pK7WIWG2 (II)). Recombinant vector $\mathrm{pEN}-\mathrm{CPi}$ transformed into $E$. coli One Shot ${ }^{\circledR}$ TOP 10 cells with variable heat shock. The colonies carrying the recombinant vector were selected by colony-PCR with specific primers SMV-CPi-F/BYMV-CPi-R and primer pair M13-F/M13-R.

Vector carrying RNAi structure containing $\mathrm{CPi}$ gene region (pK7GW-CPi [SMV-BYMV]) was created by Gateway technique (Invitrogen). Transgenic vector carrying RNAi structure containing $\mathrm{CPi}$ gene region pK7GW-CPi(SMV-BYMV) was transferred into Agrobacterium tumefaciens cells by electrical pulses $(2.5 \mathrm{kV}$, $25 \mu \mathrm{F}, 200 \Omega$ ). The colonies A. tumefaciens carrying vector pK7GW-CPi(SMV-BYMV) were selected by colony-PCR technique with specific primers SMV-CPi-F/BYMV-CPi-R.

\section{RESUlTS AND DiSCUSSION}

\section{A. Cloning and Sequence Determining of CP Gene of $S M V$, and Generating CPi (SMV-BYMV) Fragment}

From the leaves of soybean plants which were suspected to be infected by SMV, we extracted total RNA and synthesized cDNA with random primers (random hexamer primers). $C P$ gene was amplified by PCR with specific primer pair SMVcp-F/SMVcp-R and RT-PCR products obtained a very special band with the length of approximately $0.7 \mathrm{~kb}$ as expected (Fig. 2 a). PCR products were purified and inserted into cloning vector pBT_2705bp and transformed into $E$. coli DH5 $\alpha$. We selected clones carrying recombinant plasmid, extracted recombinant plasmids and checked the size of the insert by colony-PCR. The results confirmed that plasmid carrying an insert with expected size (Fig. 2 b).

The results of gene sequencing showed that $C P$ gene fragment is $720 \mathrm{bp}$ in length, encoding 240 amino acids. 
Results comparing with gene sequences $C P$ published on GenBank by using BLAST in NCBI show that sequences of $C P$ gene fragment isolated from SMV lines SL1 and SL2 are similar to that in $C P$ gene sequence (code number: X63771) on Genbank with $100 \%$ (for SMV line SL1) and $99.3 \%$ (for SMV line SL2) of similar coefficients. Results comparing two sequences of $C P$ gene fragment of lines SL1 and SL2 show there is $99.3 \%$ of similarity, and different in only 5 nucleotides (Table I).

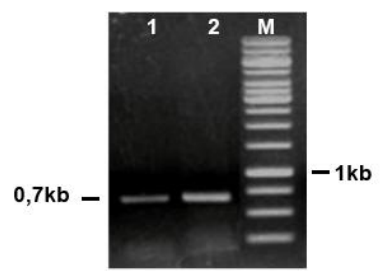

a)

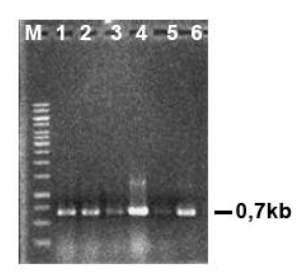

b)
Fig. 1. a) PCR products of the CP gene (cDNA) amplifed from SMV line SL1 and SL2 (1, 2: SL1, SL2; M: DNA Marker 1kb). b) Colony-PCR products of $\mathrm{CP}$ gene from colony lines $(\mathbf{1}, \mathbf{2}, \mathbf{3}$ : CP gene amplifed from SMV line SL1; 4, 5, 6: CP gene amplifed from SMV line SL2; M: DNA Marker 1kb).

TABLE I: THE DIFFERENCE BETWEEN THE SEQUENCE OF CP GENE FRAGMENT OF SMV LINE SL2 COMPARED WITH THE SMV LINE SL1 AND SEQUENCE OF CP GENE IN SMV ON GENBANK (CODE NUMBER X63771)

\begin{tabular}{cccc}
\hline \hline $\begin{array}{c}\text { Nucleotide } \\
\text { positions }\end{array}$ & X63771 & Line SL1 & Line SL2 \\
\hline 31 & $\mathrm{~A}$ & $\mathrm{~A}$ & $\mathrm{~T}$ \\
38 & $\mathrm{C}$ & $\mathrm{C}$ & $\mathrm{G}$ \\
686 & $\mathrm{~A}$ & $\mathrm{~A}$ & $\mathrm{G}$ \\
690 & $\mathrm{G}$ & $\mathrm{G}$ & $\mathrm{T}$ \\
694 & $\mathrm{~T}$ & $\mathrm{~T}$ & $\mathrm{~A}$ \\
\hline \hline
\end{tabular}

In addition, the differences between amino acid sequences were found in 5 amino acid positions $(11,13,220,230,232)$ and three positions of amino acid in poty-coat region. This result confirmed that we have successfully cloned $C P$ gene from the SMV lines SL1 and SL2.

Genomes of SMV and BYMV include genes on single-stranded RNA and the inhibition of mRNA by RNAi mechanism occurs only when there is a high homology between the siRNA and the target mRNA. On the other hand the antiviral effect of transgenic plants depends on the function of the target viral genes, so selected the genes to design the structure for RNAi technique is an important step leading to the success of this technique. We collected information about the $C P$ genes of SMV and BYMV in GenBank, used BLAST in NCBI to identify conserved regions to design $C P i$ gene region containing conserved regions of the two $C P$ genes, denoted as CPi(SMV-BYMV), with the size of 573 nucleotides.

\section{B. Designing an RNAi-Based Vector Containing CPi(SMV-BYMV) Fragment}

CPi region $C P i(S M V-B Y M V)$ was amplified by PCR with primer pair SMV-CPi-F/BYMV-CPi-R and PCR products obtained a very special band with the length of 573bp as expected (Fig. 3).

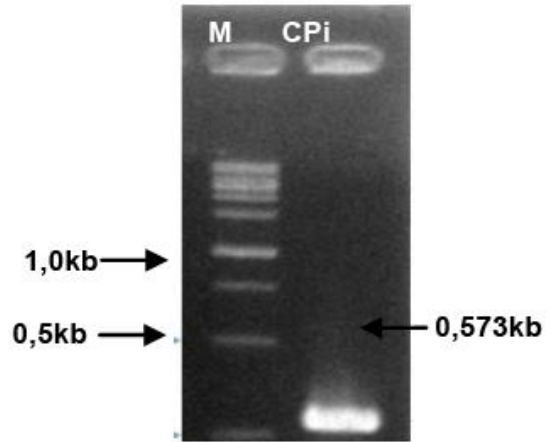

Fig. 2. PCR products of the CPi fragment amplifed from CPi (SMV-BYMV) M: DNA Marker 1kb; CPi: amplifed from CPi (SMV-BYMV)

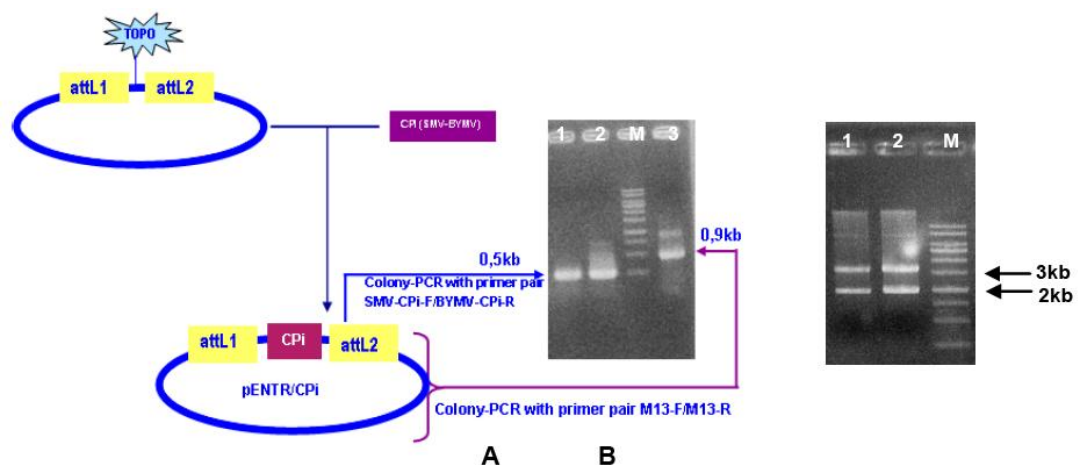

Fig. 3. A: PCR products of the CPi (SMV-BYMV) region amplifed from colonies by colony-PCR; B: Results of electrophoresis of recombinant plasmid pEN-CPi (SMV-BYMV) (M: DNA Marker 1kb; 1, 2: plasmid pEN- CPi (SMV-BYMV).

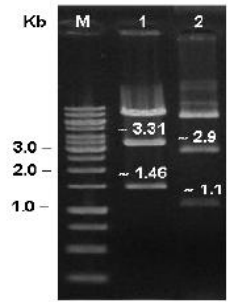

A

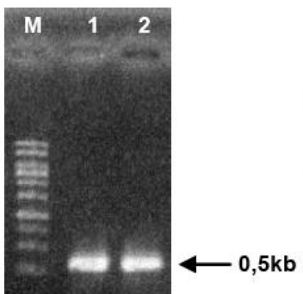

B

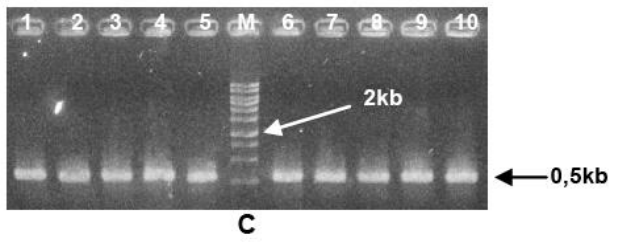

C

Fig. 4. A: Products from plasmid pK7GW-CPi(SMV-BYMV) was cut by Xba I and HindIII (M: DNA Marker 1kb; 1- vector pK7GWIWG2(II), 2pK7GW-CPi(SMV-BYMV)); B: PCR products of the CPi (SMV-BYMV) region amplifed with primer pair SMV-CPi-F/BYMV-CPi-R from plasmid pK7GW-CPi(SMV-BYMV) (M: DNA Marker 1kb; 1, 2: CPi fragment was amplified by PCR with size is $0.5 \mathrm{~kb}$ ); C: Colony-PCR products of CPi (SMV-BYMV) from colony lines (1-10: CPi (SMV-BYMV) was amplifed with size is 0.5kb; M: DNA Marker 1kb). 
PCR products were purified and inserted into cloning vector $\mathrm{pENTR}$ by $\mathrm{pENTR}^{\mathrm{TM}}$ Directional TOPO ${ }^{\circledR}$ Cloning KIT to create recombinant plasmid carrying the $\mathrm{CPi}$ (pEN-CPi (SMV-BYMV) and insert into E. coli TOP 10 One Shop ${ }^{\circledR}$. Positive colonies were checked by colony-PCR with specific primers SMV-CPi-F/BYMV-CPi-R and primer pair M13-F/M13-R (Fig. 4).

RNAi-based vector containing $C P i$ gene region, denoted as pK7GW-CPi [SMV-BYMV] was generated by adding $3 \mu \mathrm{l}$ $\mathrm{LR}$ reaction (plasmid $\mathrm{pEN}-\mathrm{CPi}$ (50-150ng), vector pK7GWIWG2(II) $(150 \mathrm{ng} / \mu \mathrm{l}), \mathrm{H}_{2} \mathrm{O}$ deionised) into $50 \mu \mathrm{l} E$. coli cell One Shot ${ }^{\circledR}$ TOP 10, with variable heat shock at $42^{\circ} \mathrm{C}$ in 45 seconds. Recombinant plasmid

pK7GW-CPi (SMV-BYMV) was checked by restriction enzymes $\mathrm{Xba} \mathrm{I}$ and colony-PCR. Recombinant vector pK7GW-CPi (SMV-BYMV) was cut by Xba I to obtain DNA fragment with the approximate size of $1.1 \mathrm{~kb}$, in which the $1.1 \mathrm{~kb}$ DNA fragment contains our target gene $\mathrm{CPi}$ (SMV-BYMV). Structure pK7GW-CPi (SMV-BYMV) was transformed into A. tumefaciens and the transformed A.tumefaciens cells were selected and checked by colony-PCR reaction with primers SMV-CPi-F/BYMV-CPi-R (Fig. 5). Positive A. tumefaciens lines were stored in glycerol at $-80^{\circ} \mathrm{C}$ to transfer into soybean cells in the future, in order to generate transgenic soybean plants that are resistant to SMV and BYMV.

\section{CONCLUSION}

SMV CP gene has been isolated and cloned from SMV cultivar SL1, SL2 (Vietnam). CP gene fragment has the size of $720 \mathrm{bp}$, encoding 240 amino acids. The two conserved regions of $C P$ gene from these two viruses (SMV and BYMV) were integrated into a $573 \mathrm{bp}$ fragment, called CPi(SMV-BYMV). Vector pK7GW-CPi (SMV-BYMV), carrying the $\mathrm{CPi}$ (SMV-BYMV) fragment in sense and antisense orientation separated by an intron, was successfully designed by Gateway technology (Invitrogen). This vector will be used to create transgenic soybean plants that are resistant to both SMV and BYMV.

\section{ACKNOWLEDGMENT}

This work has been done by the financial support of the Project B2013-TN04-05. The authors would like to express their gratefulness for the help of Key Laboratory of Gene Technology, Institute of Biotechnology, Vietnam.

\section{REFERENCES}

[1] L. L. Te and V. T. Man, Plant diseases caused by bacteria and viruses, Vietnam Educational Publisher 1999.

[2] A. Gibbs and B. Harrison, Plant virology the principles, Edward Amold, 1976.

[3] G. L. Hartman, J. B. Sinclair, and J. C. Rupe, Compendium of Soybean Diseases, Fourth Edition, Minnesota, USA: The American Phytopathological Society Press, 1999.

[4] B. James, Compendium of Soybean Diseases, "Fourth Edition, Minnesota, USA: The American Phytopathological Society Press, 1982.

[5] S. M. Hammond, E. Bernstein, D. Beach, and G. J. Hannon, "An RNA-directed nuclease mediates post-transcriptional gene silencing in Drosophila cells," Nature, vol. 404, pp. 293-296, 2000.
[6] P. D. Zamore, T. Tuschl, P. A. Sharp, and D. P. Bartel, "RNAi: double-stranded RNA directs the ATP-dependent cleavage of mRNA at 21 to 23 nucleotide intervals," Cell, vol. 101, pp. 25-33, 2000.

[7] E. Bernstein, A. A. Caudy, S. M. Hammond, and G. J. Hannon, "Role for a bidentate ribonuclease in the initiation step of RNA interference," Nature, vol. 409, pp. 295-296, 2001

[8] S. Asad, W. A. A. Haris, A. Bashir, Y. Zafar, K. A. Malik, N. N. Malik, and C. P. Lichtenstein, "Transgenic tobacco expressing geminiviral RNAs are resistant to the serious viral pathogen causing cotton leaf curl disease," Arch Virol., vol. 148, pp. 2341-2352, 2003.

[9] A. Fuentes, P. L. Ramos, E. Fiallo et al., "Intron-hairpin RNA derived from replication associated protein $\mathrm{C} 1$ gene confers immunity to Tomato yellow leaf curl virus infection in transgenic tomato plants,' Transgenic Res., vol. 15, pp. 291-304, 2006.

[10] M. Karimi, D. Inze, and A. Depicke, "Gateway-vectors for Agrobacterium-mediated plant transformation," Trends in Plant Sci., vol. 7, pp. 193-195, 2002.

[11] C. Jayaram, J. H. Hill, and W. A. Miller, "Complete nucleotide sequences of two soybean mosaic virus strains differentia ted by response of soybean containing the Rsv resistance gene," J. Gen. Virol., vol. 73, pp. 2067-2077, 1992.

[12] W.-S. Lim, Y.-H. Kim1, and K.-H. Kim, "Complete Genome Sequences of the Genomic RNA of Soybean mosaic virus Strains G7H and G5," Plant Pathol. J., vol. 19, no. 3, pp. 171-176, 2003.

[13] A. L. Haenni et al., "Potyvirus proteins: a wealth of functions," Virus Research, vol. 74, pp. 157-175, 2001.

[14] N. A. Smith, S. P. Singh et al., "Total silencing by intron-spliced hairpin RNAs," Nature, vol. 407, no. 6802, pp. 319-320, 2000.

[15] S. V. Wesley, C. A. Helliwell et al., "Construct design for efficient, effective and high-throughput gene silencing in plants," Plant J., vol. 27, no. 6, pp. 581-590, 2001.

[16] B. Kenny et al., "RNAi-Mediated Resistance to Bean golden mosaic virus in Genetically Engineered Common Bean (Phaseolus vulgaris)," Molecular Plant-Microbe Interactions, vol. 20, no. 6, pp. 717-726, 2007.

[17] H. S. Lim et al., "Soybean mosaic virus Helper Component-Protease Alters Leaf Morphology and Reduces Seed Production in Transgenic Soybean Plants," Phytopathology, vol. 97, no. 3, pp. 366-372, 2007.

[18] P. T. Van, N. V. Bac et al., "Create tobacco plants resistant to cucumber mosaic virus disease by RNAi," J. of Biotech., vol. 6, no. 4A, pp. 679-687, 2008.

[19] C. H. Ha, Đ. X. Đong et al., "Research created papaya cultivars have resistant to the ringspot diseases by application of RNAi mechanism," National Conference of plant diseases in Vietnam. Vietnam Agricultural Publisher, pp. 316-326, 2011.

[20] N. T. H. Yen, P. T. Van et al., "Create tomato line PT18 resistant yellow leaf curl virus by using RNAi," J. of Biotech., vol. 9, no. 3, pp. 333-340, 2011.

[21] J. Sambrook, E. F. Fritschi, and T. Maniatis, Molecular cloning: A laboratorymanual, New York: Cold Spring Harbor Laboratory Press, 1989.

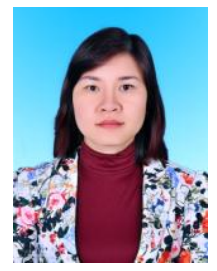

Lo Thi Mai Thu was born in Vietnam. She is a PhD student in the Department of Genetics \& Modern Biology, Thainguyen University, Vietnam. Her research interest includes plant molecular biology and RNAi technique.

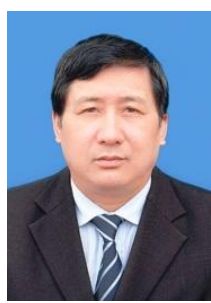

Mau Hoang Chu was born in Vietnam. He obtained his $\mathrm{PhD}$ degree in genetics at Institute of Biotechnology, VAST, Vietnam (2001). He is a professor in the Department of Genetics and modern Biology, faculty of Biology, Thainguyen University. His research interest includes molecular biology of plants, application of transgenic techniques aiming to improve the drought tolerance and improve resistance to virrus of soybean cultivars in Vietnam. Prof. Chu has some publications (Genes and drought tolerance of soybean, VNU Publishing House, Hanoi, 2011). 